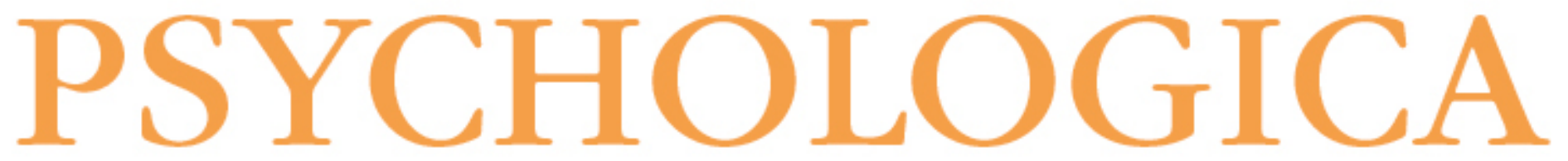

\title{
Associações entre estresse parental e práticas socioeducativas parentais em pais/mães de filhos com e sem diabetes mellitus tipo 1
}

Autor(es): $\quad$ Brito, Ariane de; Faro, André

Publicado por: Imprensa da Universidade de Coimbra

URL

persistente: URI:http://hdl.handle.net/10316.2/42371

DOI: DOI:https://doi.org/10.14195/1647-8606_60-1_6

Accessed : $\quad$ 26-Apr-2023 02:39:27

A navegação consulta e descarregamento dos títulos inseridos nas Bibliotecas Digitais UC Digitalis, UC Pombalina e UC Impactum, pressupõem a aceitação plena e sem reservas dos Termos e Condições de Uso destas Bibliotecas Digitais, disponíveis em https://digitalis.uc.pt/pt-pt/termos.

Conforme exposto nos referidos Termos e Condições de Uso, o descarregamento de títulos de acesso restrito requer uma licença válida de autorização devendo o utilizador aceder ao(s) documento(s) a partir de um endereço de IP da instituição detentora da supramencionada licença.

Ao utilizador é apenas permitido o descarregamento para uso pessoal, pelo que o emprego do(s) título(s) descarregado(s) para outro fim, designadamente comercial, carece de autorização do respetivo autor ou editor da obra.

Na medida em que todas as obras da UC Digitalis se encontram protegidas pelo Código do Direito de Autor e Direitos Conexos e demais legislação aplicável, toda a cópia, parcial ou total, deste documento, nos casos em que é legalmente admitida, deverá conter ou fazer-se acompanhar por este aviso.

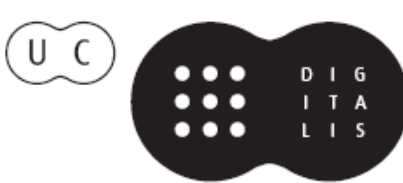


vOLUME
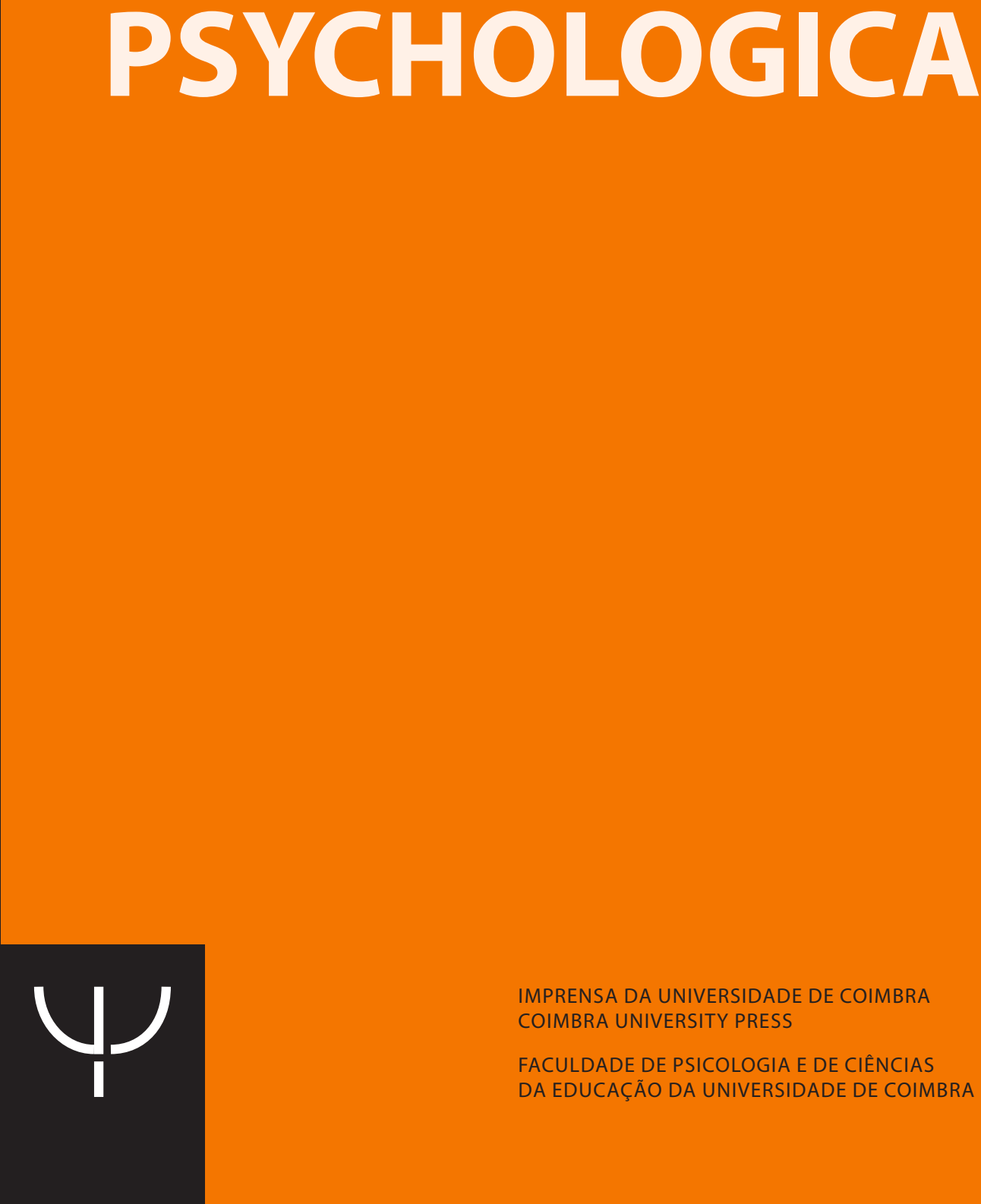

IMPRENSA DA UNIVERSIDADE DE COIMBRA

COIMBRA UNIVERSITY PRESS

FACULDADE DE PSICOLOGIA E DE CIÊNCIAS

DA EDUCAÇÃO DA UNIVERSIDADE DE COIMBRA 


\title{
Associações entre estresse parental e práticas socioeducativas parentais em pais/mães de filhos com e sem diabetes mellitus tipo 1
}

\author{
Ariane de Brito ${ }^{1}$ e André Faro ${ }^{2}$
}

\begin{abstract}
Associations between parental stress and parental socio-educational practices in fathers/mothers of children with and without type 1 diabetes mellitus
\end{abstract}

\begin{abstract}
In this research of correlational and comparative design, we examined the levels of parental stress and the parental socio-educational practices of 135 fathers/mothers of children without chronic disease (control group) and with type 1 diabetes mellitus (T1DM; clinical group). Therefore, the groups were stratified according to the reported level of parental stress (low or high stress), and significant differences were found between them, except between the control and clinical groups with low stress. The multinomial logistic regression also revealed significant differences between the groups regarding the use of socio-educational practices of affection, education and discipline, in which the fathers/mothers of the control group with low parental stress presented higher chances of using these practices. At the end, it is believed that the results can help interventions with fathers/mothers who seek to minimize parental stress and
\end{abstract}

1 Mestrado em Psicologia Social pela Universidade Federal de Sergipe (PPGPS-UFS), São Cristóvão, Sergipe, Brasil. E-mail: arianedebrito@yahoo.com.br

2 Professor Adjunto do Departamento de Psicologia (DPS) e do Programa de Pós-Graduação em Psicologia Social (PPGPS) da Universidade Federal de Sergipe (UFS), São Cristóvão, Sergipe, Brasil. E-mail: andre.faro.ufs@gmail.com 
enhance the use of positive parental practices, and promote a better glycemic control of the son/daughter with T1DM.

Keywords: parental stress; educational practices; type 1 diabetes mellitus

\section{Resumo}

Nesta pesquisa de delineamento correlacional e comparativo, examinaram-se os níveis de estresse parental e as práticas socioeducativas parentais em 135 pais/mães de filhos sem doença crônica (grupo controle) e com diabetes mellitus tipo 1 (DM1; grupo clínico). Para tanto, os grupos foram estratificados quanto ao nível de estresse parental relatado (baixo ou alto estresse) e foram constatadas diferenças significativas entre eles, exceto entre os grupos controle e clínico com baixo estresse. A regressão logística multinomial também revelou diferenças significativas entre os grupos quanto ao uso de práticas socioeducativas de afeto, educação e disciplina, onde os pais/mães do grupo controle baixo estresse parental apresentaram maiores chances de uso de tais práticas. Ao final, acredita-se que os resultados encontrados podem auxiliar intervenções com pais/mães que busquem minimizar o estresse parental e potencializar o uso de práticas parentais positivas, e promover um melhor controle glicêmico do(a) filho(a) portador(a) de DM1.

Palavras-chave: estresse parental; práticas educativas; diabetes mellitus tipo 1

O Diabetes Mellitus (DM) é uma das doenças crônicas de maior prevalência no Brasil e no mundo, considerada como problema de saúde pública mundial. Estima-se que cerca de 382 milhões de pessoas têm a doença, onde mais de 12 milhões estão no Brasil (6.2\% da população), país que ocupa o quarto lugar no ranking mundial de países com maior número de diabéticos (International Diabetes Federation, 2013).

O DM é caracterizado pelos elevados índices de glicose (açúcar) no sangue que pode levar a complicações neurológicas, renais, vasculares, entre outras (Sociedade de Endocrinologia e Metabologia, 2004). Entre os vários tipos de DM, o Diabetes Mellitus Tipo 1 (DM1) é o que mais tem crescido na população infantil (Jaser, Linsky, \& Grey, 2014; Martins, Ataíde, Silva, \& Frota, 2013), mesmo podendo ter seu início clínico em qualquer idade (Lukács, Varga, Kiss-Tóth, Soós, \& Barkai, 2014). Seu diagnóstico exige uma reorganização familiar e mudanças de hábitos alimentares e comportamentais por parte da criança e da família (Boman, Povlsen, DahlborgLyckhage, Hanas, \& Borup, 2014; Streisand, Swift, Wickmark, Chen, \& Holmes, 2005).

Por se tratar de uma doença crônica, o DM1 requer tratamento por toda a vida, aspecto que torna desgastante o cuidar de um(a) filho(a) diabético(a), pois exige 
atenção e cuidado constantes, até que o mesmo adquira capacidade de autocuidado (Jaser et al., 2014; Malerbi, 2005). Crianças diabéticas, quando possuem tratamento adequado, tendem a ser pouco afetadas pelas consequências do DM1, assemelhando-se ao desenvolvimento físico e psicológico de seus pares que não apresentam a doença (Lukács et al., 2014). Para tanto, o grau de empenho dos pais/mães torna-se essencial para o tratamento da doença, curso positivo do desenvolvimento da criança e sua qualidade de vida (Boman et al., 2014; Sundberg, Sand, \& Forsander, 2015).

Nesse contexto, os pais/mães precisam aprender as principais práticas de cuidado com o DM1, de modo a garantir um melhor tratamento para seus filhos e ensiná-los a lidar com a doença de forma positivamente adaptativa. Algumas dessas práticas incluem: identificar e lidar com sintomas (hiperglicemia e hipoglicemia) do(a) filho(a), administrar a medicação (insulina) diariamente, monitorar o nível de açúcar no sangue, fornecer alimentação balanceada e ir frequentemente ao médico. Além disso, os pais/mães precisam lidar com seus próprios sentimentos e com outras demandas peculiares da parentalidade relacionadas ou não ao DM1 (Boman et al., 2014; Martins et al., 2013; Sweenie, Mackey, \& Streisand, 2014).

Questões relativas ao tratamento e controle do DM1 ampliam demandas adaptativas e podem interferir no nível de estresse vivenciado pelos pais/mães. No contexto da parentalidade, o estresse é denominado estresse parental, compreendido como um conflito ou desequilíbrio que ocorre entre as demandas percebidas e exigidas da parentalidade e os recursos que os pais/mães possuem para lidar com elas (Park \& Walton-Moss, 2012; Skreden et al., 2012; Theule, Wiener, Tannock, \& Jenkins, 2013). O estresse parental pode afetar os pais/mães, aumentando o risco de pior saúde mental entre eles (Jaser et al., 2014), e prejudicar a capacidade em aprender habilidades de gerenciar doenças. Ademais, pode aumentar o nível de estresse vivenciado pela criança, e influir negativamente sobre a habilidade de esta lidar com adversidades ao longo de seu desenvolvimento (Streisand et al., 2005; Sweenie et al., 2014).

A literatura da área tem apontado que pais/mães de filhos com doenças crônicas vivenciam níveis de estresse parental mais elevados do que pais/mães de crianças saudáveis (Hayes \& Watson, 2013; Jaser et al., 2014; Zhang, Wei, Shen, \& Zhang, 2015). Sobre isso, Jaser et al. (2014) e Zhang et al. (2015) destacam possíveis explicações, como a sobrecarga de tarefas e exigências especiais pelas quais esses pais/ mães são expostos, podendo gerar altos níveis de estresse e tensão emocional.

Especificamente, no caso do DM1, o estresse parental tem sido apontado como um fator que pode impactar diretamente o controle do diabetes da criança (Streisand et al., 2005; Sweenie et al., 2014). Assim, torna-se relevante estudá-lo em âmbito nacional, a fim de ampliar o conhecimento e auxiliar mudanças de atitude e comportamento. 


\section{Estresse Parental e Práticas Parentais}

Considerando a parentalidade como uma experiência de duas facetas, ora gratificante, ora desgastante (Abidin, 1992; Berry \& Jones, 1995), todos os pais/mães experienciam estresse (Santos, 2008), mas o modo como a família será afetada por ele dependerá de diferentes variáveis (sociais, ambientais, etc.) e da maneira como os estressores serão percebidos durante a experiência parental. O estresse parental não é uma modificação do processo de estresse em si; a parentalidade, nesse caso, é o que define o caráter peculiar do contexto estressor.

Para Lazarus (2007), o estressor só influi sobre o organismo se o indivíduo avaliar a situação como ameaçadora ou se a percepção dos recursos que possui não for suficiente para lidar com a situação. Isto é, o estresse seria algo avaliado como sobrecarga, que excedendo os recursos ameaça o bem-estar (desfecho). Assim, a percepção dos pais/mães diante dos comportamentos da criança, do acesso aos recursos que os ajudam a atender as demandas da parentalidade, e a sensação de competência parental (Szelbracikowski, 2009), são fatores fortemente associados à necessidade de modificação do repertório adaptativo.

A correlação entre estresse parental e práticas parentais vem sendo observada em estudos brasileiros (Bérgamo \& Bazon, 2011; Minetto, Crepaldi, Bigras, \& Moreira, 2012) e outros (Guajardo, Snyder, \& Petersen, 2009; Park \& Walton-Moss, 2012; Santos, 2008). Eles têm demonstrado que níveis elevados e contínuos de estresse parental estão relacionados com o uso de práticas educativas negativas (rejeição, punição) e estilos de disciplina mais autoritário e negligente. Níveis mais baixos de estresse parental, por sua vez, aparecem associados com o uso de práticas positivas (apoio emocional) e estilos parentais autoritativos (Baumrind, 1966), os quais são caracterizados por muito controle e muito afeto (Park \& Walton-Moss, 2012; Santos, 2008). Assim, práticas parentais podem ser importantes mediadores para determinar a intensidade do estresse parental, porquanto modificam padrões comportamentais e de interações familiares podendo elevar, atenuar ou manter os níveis de estresse dos pais/mães.

No caso de crianças em condições clínicas específicas, tal qual o DM1, a doença e seu tratamento se tornam um fator adicional nos estressores/desafios adaptativos que os pais/mães enfrentam cotidianamente, como as práticas de cuidado com o diabetes, que visam garantir um melhor tratamento para seus filhos (Martins et al., 2013; Streisand et al., 2005).

A presente investigação teve como objetivo avaliar as associações entre o nível de estresse parental e as práticas socioeducativas parentais, nos grupos de pais/ mães de filhos sem doença crônica e portadores de DM1. Além disso, buscou-se identificar a intensidade do estresse parental dos grupos de pais/mães em análise 
(baixo ou alto estresse parental) e comparar os tipos de práticas socioeducativas parentais mais utilizadas entre eles.

\section{MÉTODO}

\section{Participantes}

A pesquisa realizou-se com 135 pais ou mães de pelo menos um(a) filho(a) com idade entre 3 e 15 anos, divididos em dois grupos, o GCO $(n=90)$ : grupo controle de pais/mães que obedeceram aos critérios de inclusão de ter um(a) filho(a) sem doença crônica diagnosticada e morar com ele; e o GDM $(n=45)$ : grupo clínico de pais/mães que obedeceram aos critérios de inclusão de ter um filho portador de DM1 e morar com ele.

Em relação ao grupo GCO, a idade média foi de 35.0 anos $(D P=8.60$; abrangência de 18 a 66$), 84.4 \%(n=76)$ eram mães, $43.8 \%$ possuíam ensino superior $(n=$ 39), $52.2 \%(n=47)$ trabalhavam e a renda familiar média mensal foi de R $\$ 1793.70$ $(D P=1695.79$; variando de 120.00 a 11000.00). A maioria possuía relacionamento estável $(88.9 \% ; n=80)$, média de cerca de 2 filhos $(D P=1.10$; abrangência de 1 a $7)$, não tomava remédio controlado $(87.8 \% ; n=79)$, nem possuía doença crônica $(85.6 \%$; $n=77)$. Sobre o perfil do(a) filho(a), 51.7\% $(n=46)$ eram do sexo masculino, com idade média de 7.7 anos ( $D P=3.28$; abrangência de 3 a 14$)$, frequentavam escola (97.8\%; $n=88)$ e os pais/mães os consideravam saudáveis $(93.3 \% ; n=83)$.

Já os participantes do grupo GDM tinham média de idade de 35.7 anos $(D P=$ 7.52; abrangência de 19 a 55), possuíam ensino médio (53.3\%; $n=24)$, relacionamento estável (88.9\%; $n=40)$, renda familiar média mensal de $\mathrm{R} \$ 1750.8$ ( $D P=2209.47$; variando de 400.00 a 13000.00$)$ e não trabalhavam $(55.6 \% ; n=25)$. Não possuíam doença crônica diagnosticada $(88.9 \% ; n=40)$, não tomavam remédio controlado $(86.7 \% ; n=39)$ e a média de filhos foi de $2.30(D P=1.73$; abrangência de 1 a 11$)$. Sobre o perfil do(a) filho(a), a maioria era do sexo feminino (55.6\%; $n=25$ ), com 9.7 anos de média de idade ( $D P=3.62$; abrangência de 3 a 15$)$, frequentavam escola (97.8\%; $n=88)$, e os pais/mães consideravam seus filhos saudáveis $(77.8 \% ; n=35)$. Os filhos tinham tempo médio de diagnóstico de DM1 de 41.4 meses $(D P=39.70$; abrangência de .25 a 168), aplicavam insulina em média 3 vezes por dia $(D P=1.10$; abrangência de 0 a 5), e hemoglobina glicada (HbA1c) média de 8.8 ( $D P=1.80$; abrangência de 6.2 a 14.3). A HbAlc é a medida mais importante e aceite para 
avaliar o controle glicêmico e o teste de diagnóstico para diabetes e pré-diabetes (Sociedade Brasileira de Diabetes, 2014).

A amostra foi recrutada por conveniência, na qual os participantes do GCO foram identificados nos Campus da Universidade Federal de Sergipe, Brasil (UFS) e também, por conhecidos da equipe de coleta, segundo os critérios estabelecidos da amostra. Os participantes do GDM foram recrutados na unidade do Centro de Atendimento de Especialidades Médicas (CEMAR) da cidade de Aracaju, a qual possui o Ambulatório de Endocrinologia, considerado referência para o estado de Sergipe (Brasil).

\section{Instrumentos}

Escala de Estresse Parental (EEPa) (Brito, 2016). Originalmente desenvolvida por Berry e Jones (1995), a versão brasileira é constituída por 16 itens, sendo oito questões referentes à satisfação dos pais/mães com a experiência da parentalidade $(1,3,4,5,6,11,15$ e 16) e oito relacionadas com os estressores parentais $(2,7,8$, $9,10,12,13$ e 14). O instrumento é de autorrelato e respondido em escala Likert de concordância de cinco pontos $(0=$ discordo totalmente a $4=$ concordo totalmente). No estudo de validação para o português do Brasil, a escala apresentou boa consistência interna ( $\alpha$ de Cronbach $=.81$ ) (Brito, 2016), e na presente pesquisa o alfa também foi de .81. A apuração dos resultados acontece a partir da soma dos escores de todos os itens (Mín. = 0; Máx. = 74 pontos). No entanto, na presente pesquisa a variável estresse parental foi utilizada como variável categórica/nominal e, considerando a inexistência de pontos de corte normatizados da EEPa e a distribuição normal da amostra, a média obtida pela amostra total foi utilizada como parâmetro para essa estratificação. Assim, foram criados quatro grupos quanto ao nível de estresse parental relatado em: baixo estresse parental (igual/abaixo da média) e alto estresse parental (acima da média).

Inventário de Práticas Parentais (IPP) (Benetti \& Balbinotti, 2003). Composto por 16 itens na sua versão reduzida, identifica as práticas parentais de socialização utilizadas pelos pais/mães de crianças em idade escolar. O conteúdo dos seus itens engloba três dimensões de práticas positivas: Afeto $(\alpha=.76)$, Educação $(\alpha=.82)$ e Social $(\alpha=.58)$, e uma dimensão de práticas negativas: Disciplina $(\alpha=.55)$, a qual tem sua pontuação invertida apenas para a obtenção do escore global do instrumento (Benetti \& Balbinotti, 2003). Os itens neste estudo foram respondidos a partir de uma escala Likert de frequência de cinco pontos ( $0=$ muito frequentemente a $4=$ nunca). Sobre a interpretação do escore global e por dimensão, quanto mais alto for, maior é a utilização de práticas parentais. Sua confiabilidade total se mostrou 
aceitável $(\alpha=.73)$, e por dimensão (Afeto: $\alpha=.76$; Educação: $\alpha=.63$, Disciplina $\alpha=$ .53; Social $\alpha=.50)$, apenas o alfa das dimensões Disciplina e a Social apresentaram valores um pouco abaixo do recomendado para pesquisas $(<.60)$.

Questionário de Caracterização da Amostra. Utilizado para a obtenção dos seguintes dados: (1) dados sociodemográficos dos participantes: sexo, idade, escolaridade, estado civil, renda média mensal familiar, se trabalha, se possui doença crônica diagnosticada, se toma remédio controlado e número de filhos; (2) perfil do filho (a): sexo, idade, série escolar atual e avaliação dos pais/mães do estado de saúde do(a) filho(a); e (3) dados clínicos do(a) filho(a) portador(a) de DM1 (apenas para o GDM): tempo de diagnóstico, quantidade de injeções diárias de insulina e o valor da última hemoglobina glicada mensurada (HbA1c).

\section{Procedimentos}

Os pais/mães de ambos os grupos foram informados, inicialmente, sobre o objetivo da pesquisa e convidados a participar voluntariamente, assinando o Termo de Consentimento Livre e Esclarecido (TCLE). A coleta aconteceu entre os meses de maio e dezembro de 2015. Os instrumentos foram apresentados na seguinte ordem: EEPa, IPP e Questionário de Caracterização da Amostra. O tempo médio gasto para preenchimento dos questionários foi de 15 e 20 minutos por participante dos grupos GCO e GDM, respectivamente. O presente estudo foi aprovado pelo Comitê de Ética em Pesquisa com Seres Humanos da UFS (CAAE: 36920914.9.0000.5546).

\section{Análise dos dados}

As análises foram realizadas com o auxílio do IBM SPSS Statistics 20. Inicialmente, foram feitas análises estatísticas exploratórias e descritivas para determinar a frequência (percentual e absoluta) e a amplitude de todas as variáveis do estudo. $\mathrm{O}$ estresse parental (EEPa) foi trabalhado como variável nominal (baixo estresse ou alto estresse) e a partir disso, os grupos GCO e GDM foram estratificados em dois subgrupos: GCO baixo estresse, GCO alto estresse, GDM baixo estresse e GDM alto estresse parental. Em seguida, as relações entre os quatro grupos de análise foram computadas, segundo análises descritivas e de variância (ANOVA oneway), esta última utilizada para avaliar a existência de diferenças entre as médias dos grupos. Por fim, utilizou-se a Regressão Logística Multinomial para estimar as chances de uso das práticas socioeducativas parentais por dimensão (Afeto, Educação, Disciplina e Social) a partir do grupo de pertença dos pais/mães. Na testagem do 
modelo, as dimensões Afeto, Educação, Disciplina e Social do IPP, foram utilizadas como variáveis explicativas e os grupos de análises como variáveis dependentes. Em todas as análises considerou-se o nível de significância de $p<.05$.

\section{RESULTADOS}

\section{Composição dos grupos de análise}

As médias obtidas na variável estresse parental foram examinadas. Observou-se que na amostra total a média do escore global da EEPa foi de 14.7 pontos ( $D P=$ 7.64), tendo sido, portanto, abaixo do corte de $50 \%$ da escala (32 em 64 pontos). A partir dessa média se deu a estratificação dos grupos de análises em baixo estresse (igual/abaixo da média) e alto estresse parental (acima da média). No grupo controle (GCO), 52.2\% $(n=47)$ relataram baixo estresse parental e 47.8\% $(n=43)$ alto estresse parental, já no grupo clínico $(\mathrm{GDM}), 53.3 \%(n=24)$ declararam baixo estresse e $46.7 \%(n=21)$ alto estresse parental. Assim quatro subgrupos foram formados, (1) GCO baixo estresse (34.8\%; $n=47)$, (2) GCO alto estresse (31.9\%; $n=43)$, (3) GDM baixo estresse $(17.8 \% ; n=24)$ e (4) GDM alto estresse $(15.6 \% ; n=21)$.

O perfil básico dos pais/mães dos grupos ficou caracterizado da seguinte forma: no GCO baixo estresse, $83 \%(n=39)$ eram mães, com idade média de 35.5 anos ( $D P$ = 8.65). A maioria possuía ensino médio ( $41.3 \% ; n=19)$, algum relacionamento estável $(91.5 \% ; n=43)$, renda média mensal de $\mathrm{R} \$ 1859.37$ ( $D P=1493.28)$, e média de filhos de $1.91(D P=1.06)$. Os filhos eram em grande parte do sexo masculino $(55.3 \% ; n=26)$ e com idade média de 7.4 anos $(D P=.50)$. O GCO alto estresse ficou predominantemente constituído por mães $(86 \% ; n=37)$ com idade média de 34.5 anos $(D P=8.63)$, com ensino médio $(46.5 \% ; n=20)$ e algum relacionamento estável $(86.0 ; n=37)$, renda média mensal de $\mathrm{R} \$ 1726.50(D P=1898.07)$, e média de 2 filhos $(D P=1.15)$. O perfil do(a) filho(a) indicou que 52.4\% $(n=22)$ eram do sexo feminino, com idade entre 3 e 14 anos $(M=8.1 ; D P=3.53)$.

Já o perfil do GDM baixo estresse foi de $83.3 \%(n=20)$ de mães com 36.5 anos de média de idade $(D P=6.77)$ e ensino médio $(50 \% ; n=12)$. Em geral possuíam relacionamento estável $(86 \% ; n=37)$, renda média mensal de $\mathrm{R} \$ 1828.79(D P=$ 1677.39) e 2.5 filhos em média $(D P=2.11)$. Predomínio de filhos do sexo masculino $(54.2 \% ; n=13)$, idade média de 10 anos $(D P=3.39)$, tempo médio de diagnóstico de DM1 de 36 meses $(D P=30.95)$ e média de $8.5(D P=1.87$; abrangência de 6.2 
a 14.3) no valor da última HbA1c. Por fim, o GDM alto estresse ficou composto, em maior número, por mães $(81 \% ; n=17)$ com média de idade de 35 anos $(D P=$ $8.39)$, ensino médio $(57.1 \% ; n=12), 90.5 \%$; $(n=19)$ possuía relacionamento estável, a renda média mensal de $\mathrm{R} \$ 1609.00(D P=2721.28)$. Média de filhos de 2.1 ( $D P$ $=1.16)$, os quais eram do sexo feminino (66.7\%; $n=14)$, com média idade de 9.5 anos $(D P=3.93), 47.5$ meses $(D P=47.85)$ de tempo médio de diagnóstico de DM1 e 9.1 de média $(D P=1.87$; abrangência de 6.6 a 12.8) do valor da última HbA1c.

Diferenças entre os grupos de análise

A ANOVA indicou ainda diferenças significativas entre os quatro grupos $\left[F_{(3,131)}\right.$ $=100.954 ; p<.001]$, as quais podem ser observadas na Tabela 1 . O teste Post Hoc revelou diferenças significativas entre todos os grupos $(p<.05)$, exceto entre os grupos GCO baixo estresse e o GDM baixo estresse que a diferença não foi estatisticamente significativa $(p>.05)$.

Tabela 1

Estatística Descritiva e Comparação entre os Grupos: Grupo Controle Baixo Estresse, Grupo Controle Alto Estresse, Grupo Clínico Baixo Estresse e Grupo Clínico Alto Estresse

\begin{tabular}{|c|c|c|c|}
\hline & & M (DP) & $\mathbf{p}$ \\
\hline \multirow[t]{4}{*}{ GCO baixo estresse ${ }^{a}$} & & $8.7(3.62)$ & \\
\hline & GCO alto estresse & $20.3(4.61)$ & $<.001$ \\
\hline & GDM baixo estresse & $9.0(4.69)$ & .984 \\
\hline & GDM alto estresse & $23.5(4.24)$ & $<.001$ \\
\hline \multirow[t]{4}{*}{ GCO alto estresse $^{a}$} & & $20.3(4.61)$ & \\
\hline & GCO baixo estresse & $8.7(3.62)$ & $<.001$ \\
\hline & GDM baixo estresse & $9.0(4.69)$ & $<.001$ \\
\hline & GDM alto estresse & $23.5(4.24)$ & .031 \\
\hline \multirow[t]{4}{*}{ GDM baixo estresse ${ }^{a}$} & & $9.0(4.69)$ & \\
\hline & GCO baixo estresse & $8.7(3.62)$ & .984 \\
\hline & GCO alto estresse & $20.3(4.61)$ & $<.001$ \\
\hline & GDM alto estresse & $23.5(4.24)$ & $<.001$ \\
\hline \multirow{4}{*}{ GDM alto estresse ${ }^{a}$} & & $23.5(4.24)$ & \\
\hline & GCO baixo estresse & $8.7(3.62)$ & $<.001$ \\
\hline & GCO alto estresse & $20.3(4.61)$ & .031 \\
\hline & GDM baixo estresse & $9.0(4.69)$ & $<.001$ \\
\hline
\end{tabular}

Nota: ${ }^{a}$ ANOVA (post-hoc Tukey).

Regressão Logística Multinomial entre o estresse parental e as práticas parentais

Para investigar como as práticas socioeducativas parentais se relacionam com a intensidade do estresse parental, utilizou-se a análise de regressão logística multinomial (Tabela 2). Os grupos GCO alto estresse, GDM baixo estresse e GDM alto estresse foram comparados com o grupo considerado referência, o GCO baixo 
estresse. Foram avaliados os odds ratios (OR) e os intervalos de confiança de 95\% (IC95\%), e observados os índices de Nagelkerke (variância explicada), Estatísticas de Wald (diferenças significativas na comparação entre grupos) e a Capacidade preditiva do modelo (casos corretamente preditos na amostra).

Tabela 2

Odds Ratio para os Grupos de Análise (GCO Baixo Estresse, GCO Alto Estresse, GDM Baixo Estresse e GDM Alto Estresse Parental), e seu Valor Convertido segundo Regressão Logística Multinomial tomando como Grupo de Referência o GCO Baixo Estresse Parental

\begin{tabular}{llccc}
\hline & & OR $(\mathbf{9 5 \%}$ IC) & $\begin{array}{c}\text { Conversão } \\
(\mathbf{1} / \mathbf{O R})\end{array}$ & $\boldsymbol{p}$ \\
\hline GCO alto estresse & IPP-Afeto & $.93(.72 ; 1.20)$ & - & .579 \\
& IPP-Educação & $.83(.68 ; .99)$ & 1.20 & .042 \\
& IPP-Disciplina & $.68(.56 ; .83)$ & 1.47 & $<.001$ \\
& IPP-Social & $.92(.76 ; 1.11)$ & - & .367 \\
\hline GDM baixo estresse & & & & \\
& IPP-Afeto & $.63(.47 ; .84)$ & 1.58 & .001 \\
& IPP-Educação & $1.15(.92 ; 1.44)$ & - & .210 \\
& IPP-Disciplina & $1.05(.84 ; 1.32)$ & - & .646 \\
& IPP-Social & $1.02(.82 ; 1.27)$ & - & .838 \\
\hline GDM alto estresse & & & & .033 \\
& IPP-Afeto & $0,73(0,55 ; 0,97)$ & 1,37 & .575 \\
& IPP-Educação & $1,06(0,85 ; 1,33)$ & - & .313 \\
& IPP-Disciplina & $0,89(0,71 ; 1,11)$ & - & .620 \\
\hline
\end{tabular}

O modelo testado obteve solução admissível $(p<.001)$, isto é, pelo menos uma variável explicativa foi significativa, com 33.2\% de variância explicada (Nagelkerke $=.332$ ). A capacidade preditiva total do modelo foi de $55.6 \%$ e na comparação entre grupos, as Estatísticas de Wald observadas apresentaram diferenças significativas nas dimensões Afeto $(p=.002)$, Educação $(p=.010)$ e Disciplina $(p<.001)$. A dimensão Social foi a única que não apresentou diferenças entre grupos $(p=.754)$.

Verificou-se que em comparação com o grupo de referência, o GCO alto estresse parental exibiu .83 (IC95\% = .69-.99; $p<.042)$ chance de utilizar práticas positivas de educação. Além disso, eles também exibiram .68 (IC95\% = .56-.83; $p<.001)$ chance de usar práticas negativas de disciplina. Isto é, maiores escores nas práticas de educação e disciplina são mais comuns nos pais/mães do GCO baixo estresse do que nos do grupo GCO alto estresse. Em outras palavras e com o valor de OR convertido (1/ OR), notou-se que no GCO baixo estresse, a chance de utilizar práticas de educação e de disciplina foi 1.20 e 1.47 maiores do que o GCO alto estresse, respectivamente.

O grupo GDM baixo estresse apareceu associado às práticas positivas de afeto. Eles exibiram .63 (IC95\% =.47-.83; $p=.001)$ chance de utilizar esse tipo de prática 
em sua experiência parental, ou seja, usam menos do que os pais/mães do grupo de referência. Após a conversão (1/OR), verificou-se que no GCO baixo estresse, a chance de utilizar práticas da dimensão Afeto é 1.58 maior do que no GDM baixo estresse. O mesmo ocorreu com o grupo GDM alto estresse que exibiu .73 (IC95\% $=.55-.97 ; p=.033$ ) chance de uso de práticas de afeto, isto é, os pais ou as mães do GCO baixo estresse apresentaram chance maior de 1.37 de utilizar tais práticas em comparação ao GDM alto estresse. Nesse caso, os grupos GDM, baixo e alto estresse, se diferenciam quanto à intensidade do uso dessas práticas, onde a chance de as utilizarem foi ainda menor no GDM baixo estresse.

\section{DISCUSSÃO}

A pesquisa teve como objetivo avaliar as associações entre o nível de estresse parental e as práticas socioeducativas parentais, nos grupos de pais/mães de filhos sem doença crônica e portadores de DM1. Com isso, viu-se que os pais/mães da amostra apresentaram níveis de estresse parental abaixo do corte de $50 \%$ da escala, mas mesmo assim, diferenças entre os grupos puderam ser constatadas, com exceção dos grupos GCO e GDM baixo estresse que não se diferenciaram entre si. Além disso, a regressão logística multinomial indicou diferenças significativas entre os grupos no que se refere às práticas socioeducativas de Afeto, Educação e Disciplina, onde o grupo de referência (GCO baixo estresse) apresentaram maiores chances de uso de tais práticas.

Em relação à intensidade do estresse parental encontrado neste estudo, constatou-se que a pontuação média dos pais/mães de filhos DM1 foi mais alta do que a dos pais/mães de filhos sem doença crônica. Isso corrobora achados de Hayes e Watson (2013) e Zhang et al. (2015), que também encontraram níveis mais elevados de estresse parental em contextos familiares em que há demanda clínica por parte dos filhos, ou seja, em pais/mães de crianças com alguma condição, a nível biológico, psicológico e/ou comportamental, que requerem tratamento e cuidados específicos, como por exemplo doenças crônicas, câncer, etc., quando comparados com contextos familiares que não apresentam tais condições. Tais estudos, assim, fornecem evidências de que o contexto clínico, tal como se caracteriza o contexto de pais/mães de filhos portadores de DM1, pode maximizar o estresse parental vivenciado por estes devido as demandas de cuidado impostas pelo tratamento adequado da condição clínica do(a) filho(a).

No caso do DM1, as práticas de cuidado e/ou de gerenciamento do tratamento da doença, como adequação e mudança na alimentação, monitoração da glicemia, 
administração e aplicação de insulina, costumam estar associadas com altos níveis de estresse parental, como afirmam Jaser et al. (2014). Logo, o DM1 parece criar um contexto diferenciado de estressores para a parentalidade, visto que, inicialmente, essas práticas são de responsabilidade dos pais/mães ou dos principais cuidadores, que por meio delas tentam garantir um bom controle glicêmico e uma qualidade de vida para o(a) filho(a) portador(a) de DM1 (Martins et al., 2013; Streisand et al., 2005).

Embora se saiba que o alto nível de estresse parental está mais associado com contextos clínicos (filho(a) portador(a) de DM1) quando comparados com contextos não-clínicos (filho(a) sem doença crônica), o presente estudo é o primeiro no Brasil a elucidar as diferenças entre a intensidade do estresse (baixo ou alto) em tais contextos.

Os pais/mães podem lidar de forma adaptativa, ou seja, manejando a sobrecarga ou excesso de demandas (estressores), ou lidar de forma não adaptativa, excedendo ou sobrecarregando os recursos que possuem para lidar com os estressores (demandas) da parentalidade, o que leva ao estresse parental, independentemente de o contexto ser clínico ou não clínico (Park \& Walton-Moss, 2012; Skreden et al., 2012; Theule et al., 2013). Assim, ao se avaliar as diferenças entre os grupos, viu-se que houve diferenças significativas entre os grupos controle (GCO), baixo e alto estresse parental, e entre os grupos clínicos (GDM), baixo e alto estresse parental. Esse resultado ressalta a variabilidade da forma de manejar os desafios evidenciados pelo ser pai ou ser mãe e da intensidade do estresse parental experienciado por estes no contexto controle e clínico.

Já os grupos GCO baixo estresse e GDM baixo estresse, especificamente, não se diferenciaram entre si. Estes resultados evidenciam a compreensão de que quando há um manejo adaptativo das demandas da parentalidade, ele independe de estressores próprios presentes em cada contexto analisado. Isto é, são os contextos relacionados com a criação de um(a) filho(a) sem doença crônica (não clínico) que destoam daqueles relativos à criação de um(a) filho(a) portador(a) de DM1 (clínico). Em contrapartida, com alto nível de estresse parental, o contexto pareceu interferir mais nessa relação, uma vez que o grupo GCO alto estresse e GDM alto estresse se diferenciaram significativamente um do outro. Como dito anteriormente, o facto de ambos os grupos de pais ou mães terem relatado alto estresse parental, os do GDM obtiveram níveis mais elevados em comparação com os do GCO, resultado que é consistente com estudos anteriores que comparam pais de filhos com e sem alguma doença crônica (Hayes \& Watson, 2013; Jaser et al., 2014; Zhang et al., 2015).

Sobre o modelo de regressão logística multinomial, constatou-se que as práticas parentais de afeto, educação e disciplina exibiram relação com a intensidade de estresse parental nos contextos controle (filhos sem doença crônica) e clínico (filhos portadores de DM1). Quanto ao uso de tais práticas, os pais/mães dos gru- 
pos clínicos (GDM baixo estresse e GDM alto estresse) utilizam práticas positivas [educação: "ajudar com os deveres escolares do(a) filho(a)] e negativas [disciplina: "dar palmadas no filho(a) quando só falar não é suficiente"] tanto quanto os pais/ mães dos grupos controle (GCO baixo estresse e GCO alto estresse). No entanto, especialmente nas práticas de afeto, como "beijar e abraçar o(a) filho(a)", os grupos clínicos usam menos do que o GCO baixo estresse. Observou-se, então, que as demandas próprias do DM1 presentes na rotina parental podem ter afetado esse resultado, havendo a necessidade desses pais/mães desenvolverem mais práticas de afeto, já que elas maximizam a interação familiar.

As práticas parentais de educação e de disciplina foram mais usadas pelos pais ou mães do GCO baixo estresse, sugerindo que esse grupo consegue manejar de forma mais adaptativa demandas da parentalidade (isto é, enfrentamento de estressores cotidianos), e que, portanto, fazem uso de práticas positivas e negativas de modo aproximado. Considerando a literatura a respeito das práticas parentais, o uso de práticas tanto negativas quanto positivas seria característico do estilo parental denominado autoritativo (Baumrind, 1966), no qual há muito controle e muito afeto (Park \& Walton-Moss, 2012), o que remete ao maior uso de práticas de educação (positiva) e também de disciplina (negativa) frente às demandas da parentalidade.

A mesma lógica pode servir para explicar o fato do grupo GCO alto estresse da presente pesquisa utilizarem menos tais práticas (educação e disciplina). Assim, esse resultado sugere que o alto estresse pode interferir, nesse caso, não só na diminuição do uso de práticas positivas [práticas de educação, por exemplo, “ajudar com os deveres escolares do(a) filho(a)"], mas também na redução do uso de práticas negativas [práticas de disciplina, por exemplo, "gritar com o(a) filho(a) quando ele(a) faz alguma coisa errada"]. Esse resultado, entretanto, difere do que se costuma encontrar na literatura da área, em que pais/mães com alto e contínuos índices de estresse parental aparecem correlacionados com o estilo de disciplina parental mais autoritário. Esse estilo costuma ser caracterizado, principalmente, pelo uso de práticas educativas negativas, tais como controle e punição nas interações com os filhos, e maior probabilidade de ocorrerem abusos físicos e de manter o comportamento indesejado da criança (Bergámo \& Barzon, 2011; Guajardo et al., 2009; Park \& Walton-Moss, 2012; Szelbracikowski, 2009).

Já no caso do uso das práticas parentais de afeto, os dados desta investigação demonstraram que ele é influenciado tanto pelo contexto parental analisado (filhos sem doença crônica e filhos com DM1), quanto pela intensidade do estresse parental relatado pelos pais/mães. Acredita-se que isso tenha ocorrido porque os grupos clínicos GDM, baixo e alto estresse, apresentaram menores chances de uso dessas práticas, como "elogiar o(a) filho(a)", em comparação com o GCO baixo estresse (grupo de referência). 
Ainda que os pais ou mães dos grupos clínicos (GDM) façam uso de todas as práticas socioeducativas parentais avaliadas, assim como ocorreu com os pais/ mães dos grupos controle (GCO), os primeiros fazem menos uso de práticas, sejam elas positivas (afeto e educação) ou negativas (disciplina). Isso pode ser pensado a luz das condições específicas presentes nos diferentes contextos e no perfil dos pais e mães participantes.

É comum, a título de exemplo, que pais/mães de crianças portadores de DM1 apresentem práticas de superproteção com os filhos (Martins et al., 2013), o que pode interferir em vários aspectos do desenvolvimento infantil como, por exemplo, na autonomia da criança, fazendo despertar sentimentos de dependência, instabilidades emocionais e insegurança nos filhos. Essa superproteção poderia justificar a diminuição de práticas parentais de disciplina, por exemplo, já que como forma de proteção pais e mães exigem menos dos filhos e/ou realizam atividades que por ora deveriam ser feitas pela própria criança (Souza, 2013). Por fim, vale destacar que na amostra pesquisada, as práticas socioeducativas parentais da dimensão Social [por exemplo: "participar de jogos/atividades com o(a) filho(a)]" não apresentaram diferenças estatisticamente significativas entre os grupos analisados, o que nos leva a crer que práticas de lazer são comuns em pais ou mães de filhos sem doença crônica e de filhos com DM1.

\section{Limitações do estudo e considerações finais}

Alguns aspectos da pesquisa limitam a generalização dos resultados aqui encontrados. O primeiro deles se refere a amostra total, que pode ser considerada pequena $(N=135)$, visto que houve ainda a divisão por grupos. $\mathrm{O}$ tamanho do grupo de pais/mães de filhos DM1 foi o menor $(n=45)$, o que pode ter por ora influído nas análises realizadas. Com amostras maiores e mais homogêneas os efeitos dos resultados encontrados poderiam ter sido diferentes. Ainda assim, vale lembrar que o recrutamento de grandes amostras dentro de qualquer população com doença crônica é muitas vezes bastante difícil, principalmente, em se tratando do público de pais/mães de uma população tão específica que é a de crianças com DM1.

Outro aspecto limitante diz respeito ao modelo testado. Apesar de ter sido aceitável nesta primeira tentativa de explicação das associações entre práticas socioeducativas parentais e a intensidade do estresse parental nos grupos analisados, acredita-se que o modelo futuramente deva ser testado mediante a inclusão de novas variáveis/construtos para análise do desfecho, tais como o controle glicêmico do(a) filho(a) portador(a) de DM1. Dado que os principais preditores do estresse parental são os aspectos do ambiente familiar, as características dos pais e dos filhos e os eventos de vida estres- 
santes (Abidin, 1992; Costa, 2011), associações com variáveis como estas (perfil dos pais, dos filhos, características clínicas dos filhos portadores de DM1, etc.), podem, em futuras investigações, contribuir para ampliação e debates dos presentes achados.

Além disso, a confiabilidade das dimensões Disciplina e Social do IPP podem ser consideradas baixas em relação ao recomendado. Acredita-se que o número de itens e o tamanho da amostra possam ter influenciado esse resultado. Ainda assim, para futuras pesquisas recomenda-se atenção particular nos itens que compõe essas dimensões (Benetti \& Balbinotti, 2003) ou ainda, a utilização de outras medidas de práticas parentais que se mostrem aceitáveis quanto a sua confiabilidade na amostra utilizada.

Contudo, sabe-se que abordar fatores que contribuem para a promoção de interações mais positivas entre pais/mães e filhos(as), torna-se cada vez mais relevante, principalmente quando há a presença do estressor "doença crônica do(a) filho(a)". O comportamento parental, suas práticas e o estresse parental, podem ser bons meios de investigação para se pensar em intervenções que contribuam positivamente para os relacionamentos entre pais e filhos e uma melhor adesão ao tratamento e controle glicêmico do(a) filho(a) portador(a) de DM1.

\section{REFERÊNCIAS}

Abidin, R. R. (1992). The determinants of parenting behavior. Journal of Clinical Child Psychology, 21(4), 407-412. doi: 10.1207/s15374424jccp2104_12

Baumrind, D. (1966). Effects of authoritative parental control on child behavior. Child Development, 37(4), 887-907. doi: 10.2307/1126611

Benetti, S. P. C., \& Balbinotti, M. A. A. (2003). Elaboração e estudo de propriedades psicométricas do Inventário de Práticas Parentais. Psico-USF, 8(2), 103-113. doi: 10.1590/S1413-82712003000200002

Bérgamo, L. P. D., \& Bazon, M. R. (2011). Abuso físico infantil: Analisando o estresse parental e o apoio social. Psicologia: Teoria e Pesquisa, 27(1), 13-21. doi: 10.1590/S0102-37722011000100003

Berry, J. O., \& Jones, W. H. (1995). The Parental Stress Scale: Initial psychometric evidence. Journal of Social and Personal Relationships, 12(3), 463-472. doi: 10.1177/0265407595123009

Boman, A., Povlsen, L., Dahlborg-Lyckhage, E., Hanas, R., \& Borup, I. K. (2014). Fathers of children with type 1 diabetes: Perceptions of a father's involvement from a health promotion perspective. Journal of Family Nursing, 20(3), 337-354. doi: 10.1177/1074840714539190

Brito, A. (2016). Estresse parental e práticas socioeducativas parentais em pais/mães de filhos com e sem diabetes mellitus tipo 1 (Dissertação de mestrado não publicada). Universidade Federal de Sergipe, São Cristóvão, Brasil. Consultado em https://bdtd.ufs.br/bitstream/tede/2887/2/ ARIANE_BRITO.pdf

Costa, C. A. C. (2011). Indutores de stress parental (Dissertação de mestrado não publicada). Universidade Fernando Pessoa, Porto. Consultado em http://bdigital.ufp.pt/bitstream/10284/1977/1/ DM_13655.pdf 
Guajardo, N. R., Snyder, G., \& Petersen, R. (2009). Relationships among parenting practices, parental stress, child behaviour, and children's social-cognitive development. Infant and Child Development, 18(1), 37-60. doi: 10.1002/icd.578

Hayes, S. A., \& Watson, S. L. (2013).The impact of parenting stress: A meta-analysis of studies comparing the experience of parenting stress in parents of children with and without autism spectrum disorder. Journal of Autism and Developmental Disorders, 43(3), 629-642. doi: 10.1007/s10803-012-1604-y

IBM Corp. (2011). IBM SPSS Statistics for Windows (Version 20.0). Armonk, NY: IBM Corp.

International Diabetes Federation (2013). IDF Diabetes Atlas (6 ${ }^{\text {th }}$ ed.). Consultado em http://www. diabetesatlas.org/resources/previous-editions.html

Jaser, S. S., Linsky, R., \& Grey, M. (2014). Coping and psychological distress in mothers of adolescents with type 1 diabetes. Maternal and Child Health Journal, 18, 101-108. doi: 10.1007/s10995-013-1239-4

Lazarus, R. S. (2007). Stress and emotion: A new synthesis. In A. Monat, R. S. Lazarus, \& G. Reevy (Eds.), The Praeger Handbook on Stress and Coping (pp. 33-52). London: Praeger.

Lukács, A., Varga, B., Kiss-Tóth, E., Soós, A., \& Barkai, L. (2014). Factors influencing the diabetesspecific health-related quality of life in children and adolescents with type 1 diabetes mellitus. Journal of Child Health Care, 18(3), 253-260. doi: 10.1177/1367493513486964

Malerbi, F. E. K. (2005). Ajustamento emocional à doença por pais de crianças e adolescentes portadores de diabetes. Psicologia Revista, 14(1), 75-108. Consultado em http://revistas.pucsp.br/ index.php/psicorevista/article/view/18129/13493

Martins, E. M. C. S., Ataíde, M. B. C., Silva, D. M. A., \& Frota, M. A. (2013). Vivência de mães no cuidado à criança diabética tipo 1. Revista da Rede de Enfermagem do Nordeste, 14(1), 42-49. doi: 10.15253/rev\%20rene.v14i1.3323

Minetto, M. F., Crepaldi, M. A., Bigras, M., \& Moreira, L. C. (2012). Práticas educativas e estresse parental de pais de crianças pequenas com desenvolvimento típico e atípico. Educar em Revista, 43, 117-132. doi: 10.1590/S0104-40602012000100009

Park, H., \& Walton-Moss, B. (2012). Parenting style, parenting stress, and children's health-related behaviors. Journal of Developmental \& Behavioral Pediatrics, 33(6), 495-503. doi: 10.1097/ DBP.0b013e318258bdb8

Santos, V. A. B. (2008). Stress parental e práticas parentais em mães de crianças com perturbação de hiperactividade com défice de atenção (Dissertação de mestrado não publicada). Universidade de Lisboa, Lisboa. Consultado em http://repositorio.ul.pt/bitstream/10451/695/1/17675_Dissertacao_Mestr_Integ_Psic_Vanessa_Santos.pdf

Skreden, M., Skari, H., Malt, U. F., Pripp, A. H., Björk, M. D., Faugli, A., Emblem, R. (2012). Parenting stress and emotional wellbeing in mothers and fathers of preschool children. Scandinavian Journal of Public Health, 40(7), 596-604. doi: 10.1177/1403494812460347

Sociedade Brasileira de Diabetes (2014). Conduta terapêutica no diabetes tipo 2: Algoritmo SBD 2014. Posicionamento Oficial SBD no 01/2014. Consultado em http://www.sgc.goias.gov.br/upload/ arquivos/2014-05/posionamento-dm2-2014.pdf

Sociedade de Endocrinologia e Metabologia (2004). Diabetes mellitus: Classificação e diagnóstico. Consultado em http://diretrizes.amb.org.br/_BibliotecaAntiga/diabetes-mellitus-classificacao-e-diagnostico.pdf

Souza, L. M. (2013). Percepção parental sobre a infância: Um estudo de crianças com e sem diabetes (Monografia). Faculdade de Psicologia do Centro Universitário de Brasília, Brasília. Consultado em http:// repositorio.uniceub.br/bitstream/235/4526/1/Monografia\%20Let\%C3\%ADcia\%20Medeiros.pdf 
Streisand, R., Swift, E., Wickmark, T., Chen, R., \& Holmes, C. S. (2005). Pediatric parenting stress among parents of children with type 1 diabetes: The role of self-efficacy, responsibility, and fear. Journal of Pediatric Psychology, 30(6), 513-521. doi: 10.1093/jpepsy/jsi076

Sundberg, F., Sand, P., \& Forsander, G. (2015). Health-related quality of life in preschool children with type 1 diabetes. Diabetic Medicine, 32(1), 116-119. doi: 10.1111/dme.12557

Sweenie, R., Mackey, E. R., \& Streisand, R. (2014). Parent-child relationships in type 1 diabetes: Associations among child behavior, parenting behavior, and pediatric parenting stress. Family Systems Health, 32(1), 31-42. doi: 10.1037/fsh0000001

Szelbracikowski, A. C. (2009). Um estudo sobre crianças pré-escolares socialmente competentes e crianças pré-escolares com comportamentos externalizados no contexto familiar (Tese de doutorado não publicada). Universidade de Brasília, Brasília.

Theule, J., Wiener, J., Tannock, R., \& Jenkins, J. M. (2013). Parenting stress in families of children with ADHD: A meta-analysis. Journal of Emotional and Behavioral Disorders, 21(1), 3-17. doi: $10.1177 / 1063426610387433$

Zhang, Y., Wei, M., Shen, N., \& Zhang, Y. (2015). Identifying factors related to management during the coping process of families with childhood with chronic conditions: A multi-site study. Journal of Pediatric Nurse, 30(1), 160-173. doi: 10.1016/j.pedn.2014.10.002 